\title{
Analysis of Salmonella Contaminated Beef Odor Using an Electronic Nose
}

\author{
Giyoung Kim*, Kangjin Lee, Jae Yong Son, and Hak-Jin Kim \\ National Academy of Agricultural Science, RDA, Suwon 441-857, Korea \\ ${ }^{1}$ Department of Bio-Industrial Machinery Engineering, Pusan National University, Miryang 627-706, Korea
}

\begin{abstract}
An electronic nose was used to identify Salmonella contamination on beef based on odors. To detect pathogen contamination of beef, $100 \mu \mathrm{L}$ of $10^{5} \mathrm{CFU} / \mathrm{g}$ Salmonella Enteritidis or Salmonella Typhimurium cell suspensions were spiked onto $5 \mathrm{~g}$ beef sirloin samples in individual vials. Odor changes over time were then measured and analyzed using an electronic nose system to identify pathogen contamination. In principle, the electronic nose system based on a surface acoustic wave (SAW) detector produced different frequency responses depending on the time and amount of each chemical. Multivariate analysis of the odor data was conducted to detect Salmonella contamination of beef. Salmonella odors were successfully distinguished from uncontaminated beef odors by principal component analysis (PCA). The PCA results showed that Salmonella contamination of beef could be detected after $4 \mathrm{~h}$ of incubation. The numbers of cells enumerated by standard plate count after $4 \mathrm{~h}$ of inoculation were $2 \times 10^{6} \mathrm{CFU} / \mathrm{g}$ for both Salmonella Enteritidis and Salmonella Typhimurium.
\end{abstract}

Key words: Electronic nose, Salmonella, Odor analysis, PCA

\section{Introduction}

Increasing public awareness for food safety makes quality evaluation of meat, poultry, and their products more important. Since olfaction is important in evaluation of the quality and safety of foods, many efforts have been made to develop an instrument that mimics the olfactory system in the nose. The instrument that operates on the similar principle as human nose is called an electronic nose.

Electronic noses are based on the array of artificial receptors. Although they differ from each other in the operating principle, the number of sensors, and the sensitivity and selectivity, their function is same. They detect volatile or semi-volatile vapors by means of sensors and send signals to recognition units to interpret the signal pattern. A typical electronic nose system consists of a chemical sensor array, electronics, pumps, an air conditioner, a flow controller, and software for hardware controlling and data processing.

Electronic nose systems have several advantages over

\footnotetext{
*Corresponding author: Giyoung Kim, Department of Agricultural Engineering, National Academy of Agricultural Science, RDA, Suwon 441-857, Korea. Tel: 82-31-290-1899, Fax: 8231-290-1900, E-mail: giyoung@korea.kr
}

conventional analyses of volatile compounds by traditional analytical techniques and by human sensory panel methods. The conventional analytical techniques such as gas chromatography/mass spectrometry (GC/MS) allow high accuracy and low detection limits, but are expensive and time and labor consuming. While sensory methods are subjective and have poor reproducibility. Even though electronic noses are not complete replacement of the sensory methods or the analytical techniques, they combine advantages of the two disciplines. These electronic nose systems are objective and produce reproducible analysis resulting in a relatively short time.

Electronic noses have been used in the quality and freshness evaluation of foods which is related to the food safety. O'Connell et al. (2001), who used a simple electronic nose to determine the fish freshness of Argentinean hake. They could identify between fresh and old samples in terms of storage days in a refrigerator. Storey et al. (2001) applied solid state gas sensors to the measurement of the trimethylamine and dimethylamine generated from spoilage of fish and fish products. Grigioni et al. (2000) identified warmed-over flavour (WOF) aroma in low temperature-long time processed meat. The WOF odor could be classified by using an electronic nose based on conduction polymer sensors. Natale et al. (2001) combined two electronic nose 
systems to increase the evaluation performance of codfish freshness. Barbri et al. (2008) successfully assessed freshness of sardines stored at $4^{\circ} \mathrm{C}$ with an electronic nose composed of six tin oxide gas sensors and a multi-class classifier model. Detection of bacteria spoilage of foods using an electronic nose was also investigated (Arnold and Senter, 1998; Blixt and Borch, 1999; Keshri et al., 1998; McEntegart et al., 2000). By using an electronic nose based on metal oxide semiconductor (MOS) sensors, Rossi et al. (1995) could correctly classified $90.5 \%$ of odors generated by bacteria grown on agar medium. The researchers also detected and classified pathogenic strains sometimes found in meat product by using the electronic nose (Rossi et al., 1996). Dutta et al. (2002) used an electronic nose comprising an array of thirty-two gas sensors to identify six species of bacteria responsible for eye infections. They could classify the bacteria with $96 \%$ and $98 \%$ of accuracy with Self Organizing Map and Radial basis function network, respectively.

The goal of this study was to investigate the feasibility of using an electronic nose for the detection of Salmonella contamination in beef. Contamination of pathogenic bacteria onto beef was measured by an electronic nose and analyzed by multivariate data analysis technique. Principal component analysis (PCA) was used to reduce the dimension of the odor chromatogram and to determine odor of Salmonella Enteritidis or Salmonella Typhimurium contaminated beef. Also, qualitative analysis of the volatile organic compound isolated from the headspace of Salmonella Enteritidis or Salmonella Typhimurium was performed with a mass spectrometer.

\section{Materials and Methods}

\section{Beef sample preparation}

Beef sirloins were obtained from a local market. The sirloins were cut into $20 \mathrm{~mm}$ thick steak and packed in sanitized vinyl bags. The sample bags were stored in a refrigerator at $5^{\circ} \mathrm{C}$ before experiments. Only the muscle part of the meats was used for odor measurements to acquire uniform odors.

\section{Bacteria and enrichment media}

Salmonella Enteritidis ATCC 14028 and Salmonella Typhimurium ATCC 13076 were acquired from American Type Culture Collection (ATCC). All fresh cultures for experiments were obtained by inoculating freezedried cultures into enrichment broth and incubating them at $37^{\circ} \mathrm{C}$ for $24 \mathrm{~h}$. For both Salmonella Enteritidis and
Salmonella Typhimurium ( $S$. Typhimurium) cell enrichment, brain heart infusion (BHI) broth (Difco Laboratories, USA) was used.

\section{Electronic nose system}

Electronic nose system used in this study was zNose 7100 (Electronic Sensor Technology, USA). The zNose is based on gas chromatography (GC) and a surface acoustic wave (SAW) detector. Unlike conventional GC, the one in the zNose is a portable GC, which uses a direct heated $1 \mathrm{~m}$ capillary column to make the system smaller and to achieve fast analysis.

In principle, odors or vapors from samples enter the system through a temperature controlled inlet and are preconcentrated for a specific time in a Tenax trap. The preconcentrated vapors are swept by helium carrier gas into the temperature programmable column. The column temperature is programmed to follow a linear rise to its maximum temperature. The linear temperature rise causes the different chemical components to be released and travel through the column with a different relative velocity.

The SAW detector consists of an uncoated acoustic resonator bonded to a Peltier thermoelectric heat pump to heat or to cool the quartz substrate. The vapors dispersed from the column are passed to the SAW detector, which produces frequency response depends on the time and amount of each chemical. Control software of the electronic nose system displays the sensor frequency and derivative-of-frequency which is equivalent to a conventional chromatogram.

For testing the beef samples, the column was ramped from 30 to $150^{\circ} \mathrm{C}$ at $5^{\circ} \mathrm{C} / \mathrm{sec}$ and the detector was held at $30^{\circ} \mathrm{C}$ during the analysis. The sampling time was $10 \mathrm{sec}$ and the same conditions were maintained during the experiments.

\section{Odor analysis procedure}

To measure the headspace chemistry of beef samples for detection of Salmonella contamination, each of $5 \mathrm{~g}$ aliquots was cut from the stored beef specimen and placed into a $40 \mathrm{~mL}$ septa-sealed vial. The beef aliquot was inoculated using $100 \mu \mathrm{L}$ of $10^{5} \mathrm{CFU} / \mathrm{g}$ Salmonella Enteritidis or Salmonella Typhimurium cell suspensions. And then, the vial was capped and placed into a dry bath at $37^{\circ} \mathrm{C}$ until odor measurements. Vapor samples were collected and preconcentrated by inserting a side-ported sampling needle through the septa at every $1 \mathrm{~h}$. The preconcentrated vapors were then injected and analyzed by the electronic nose as shown in Fig. 1. The entire mea- 


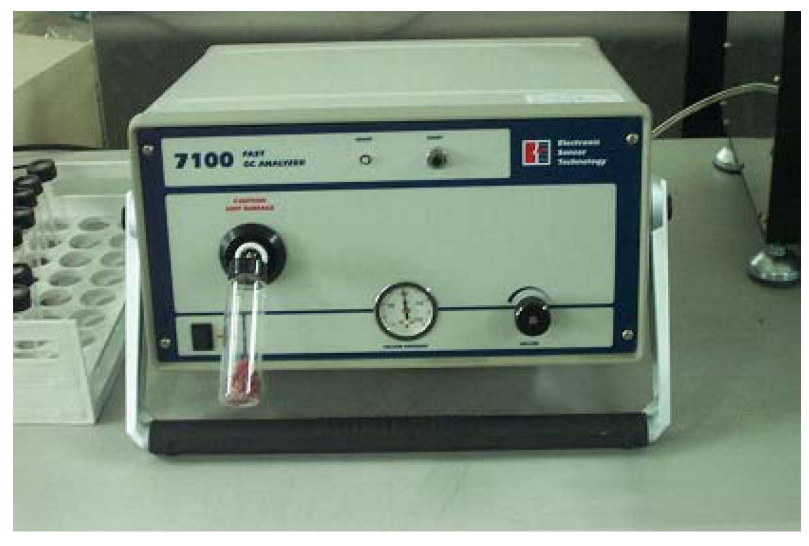

Fig. 1. The SAW based an electronic nose system for detecting Salmonella odor from beef sample.

suring process took approximately 1 min per sample.

\section{Odor data analysis}

Odor analyses were performed using multivariate analysis techniques. A PCA technique was used to reduce the dimension of the odor chromatogram. Odors were classified by PCA to differentiate odor changes resulting from Salmonella growth. For the multivariate analysis, Unscrambler 9.7 (CAMO, Norway) was used.

\section{Analysis of volatile metabolites}

The headspace volatile compounds produced by Salmonella growth were collected using solid-phase microextraction (SPME) fibers $(50 / 30 \mu \mathrm{m}$ DVB/CAR/PDMS, $65 \mu \mathrm{m}$ PDMS/DVB) and analyzed by gas chromatography-mass spectrometry (GC/MS, QP 5050A, Shidmazu, Japan) operated in the electron-ionisation (EI) mode. Headspace vapors were collected from BHI broth instead of beef to reduce the odor variation caused by beef itself. Salmonella Enteritidis or Salmonella Typhimurium was incubated in brain heart infusion (BHI) broth at $37^{\circ} \mathrm{C}$ for $24 \mathrm{~h}$. And then, the absorption of the compounds to the SPME fiber was performed at $37^{\circ} \mathrm{C}$ for $30 \mathrm{~min}$. The desorption time from SPME fiber for GC/MS measurement was 3 min with an inlet temperature of $250^{\circ} \mathrm{C}$. The GC column was an $30 \mathrm{~m} \times 0.25 \mathrm{~mm}$ (id), $0.25 \mu \mathrm{m}$ film thickness ValcoBond VB-5 (Valco Instruments, Switzerland). Helium was used as the carrier gas with flow rate of $1.0 \mathrm{~mL} / \mathrm{min}$. The GC method used following conditions: initial oven temperature of $40^{\circ} \mathrm{C}$ for $5 \mathrm{~min}$, increasing to $250^{\circ} \mathrm{C}$ at $15^{\circ} \mathrm{C} / \mathrm{min}$, and then held at $250^{\circ} \mathrm{C}$ for $10 \mathrm{~min}$; detector temperature $270^{\circ} \mathrm{C}$.

\section{Results and Discussion}

Detection of Salmonella contaminated beef was tested by analyzing the responses of the electronic nose to different growth stages of $S$. Enteritidis or $S$. Typhimurium. $S$. Enteritidis or $S$. Typhimurium cells were spiked onto the beef and the odors were measured with the electronic nose. Chromatographic analysis of odors showed distinctive patterns for each bacterium. Fig. 2 and Fig. 3 show the response curves of the electronic nose to different growth stages of $S$. Enteritidis or $S$. Typhimurium, respectively.

At an early growth stage of $1 \mathrm{~h}$, there was no noticeable peak and no distinctive odor difference between responses of different Salmonella strains. As growth time increases, peaks were emerged at a certain retention time (RT). The highest peak appeared to be at RTs of 23.8-24.8 for the Salmonella contaminated beef. Peaks were emerged at 2 $\mathrm{h}$ after incubation. However, intensities of response peaks

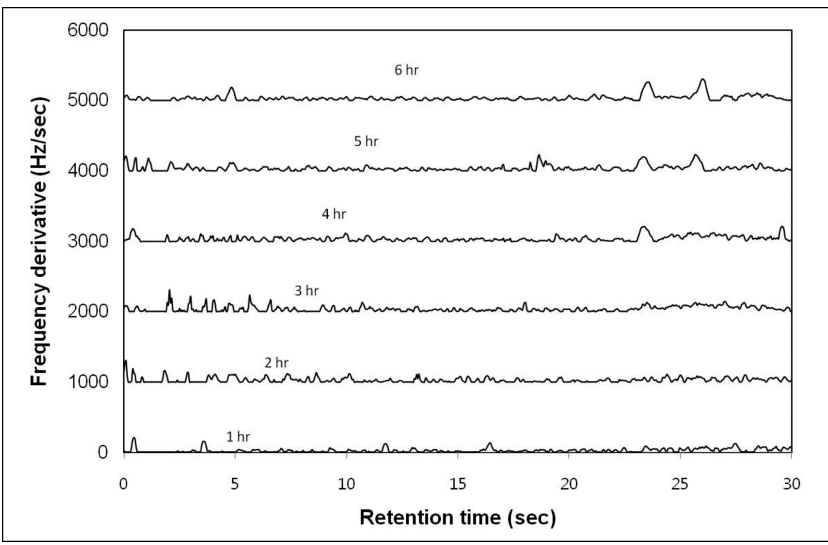

Fig. 2. Response curves of the electronic nose on growth of $S$. Enteritidis spiked onto beef.

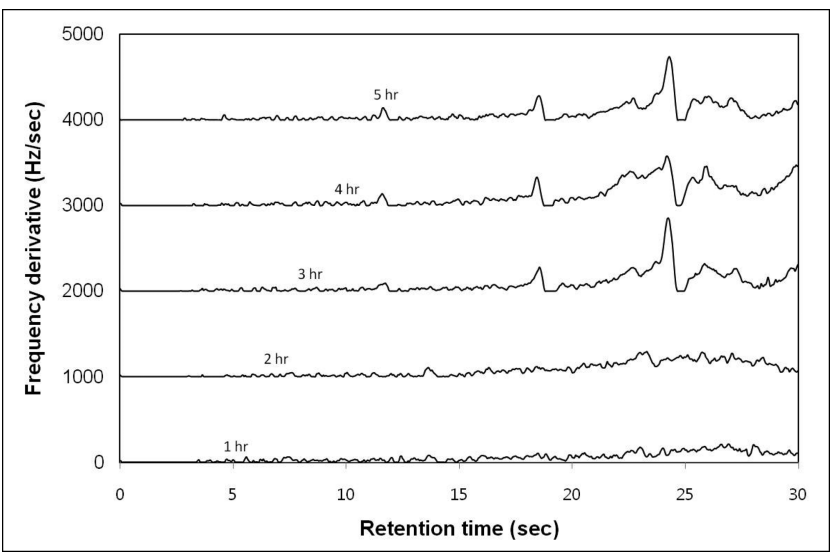

Fig. 3. Response curves of the electronic nose on growth of $S$. Typhimurium spiked onto beef (Curves marked 2, 3, 4 , and $5 \mathrm{~h}$ were offset for clarity). 
were weak because the emission of volatile compounds was not strong at such an early growth stage. The intensities were increased with increasing the growth time because more volatile compounds were produced from the metabolism of the bacteria.

PCA was performed to classify $S$. Enteritidis or $S$. Typhimurium odor from uncontaminated beef odor. Fig. 4 and Fig. 5 show PCA score plots of $S$. Enteritidis odor and $S$. Typhimurium odor classification, respectively. At relatively early growth stages (upto $3 \mathrm{~h}$ of incubation), $S$. Enteritidis contamininated beef odors were positioned near the odors of uncontaminated beef in the score plot (Fig. 4). As growth time increases (4-6 h of incubation), $S$. Enteritidis contamininated beef odors were departed away from the odors of uncontaminated beef. PCA score plot shows that the classification $S$. Enteritidis contamininated beef odor was mainly explained by PC1 (56.8\%).

$S$. Typhimurium contamininated beef odor was more distinctive than $S$. Enteritidis contamininated beef odor. $S$. Typhimurium contamininated beef odor could be differentiated from the odor of uncontaminated beef even at relatively early growth stages (Fig. 5). The odor difference between $S$. Typhimurium contamininated and uncon-

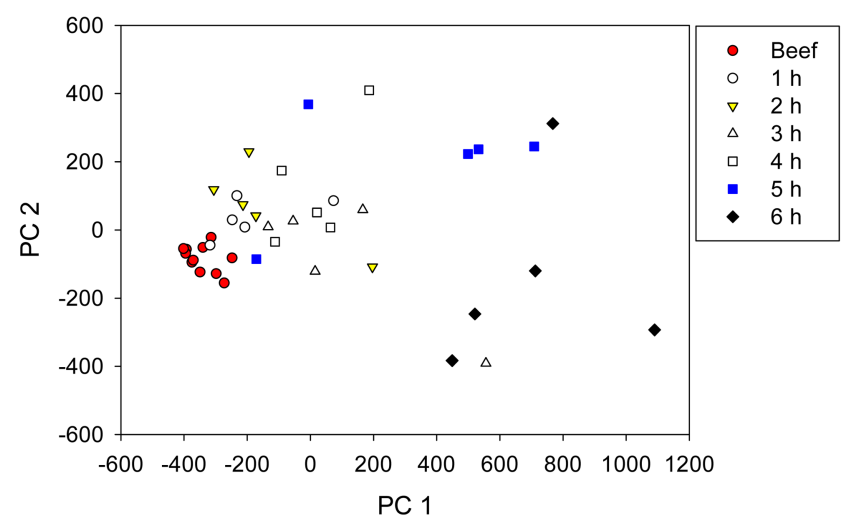

Fig. 4. PCA score plot for the classification of $S$. Enteritidis.

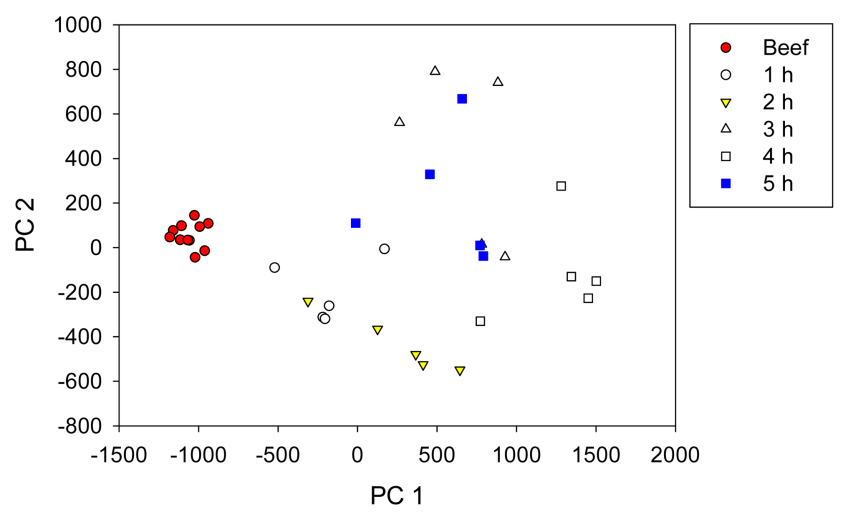

Fig. 5. PCA score plot for the classification of $S$. Typhimurium. taminated beef was clear after $3 \mathrm{~h}$ of incubation. The classification of $S$. Typhimurium contamininated beef odor was also mainly explained by PC1 (73.7\%).

By using PCA, Salmonella contaminated beef odor was successfully differentiated from uncontaminated beef odor after $4 \mathrm{~h}$ of incubation (Fig. 6). The number of cells enumerated by standard plate count for beef samples incubated $4 \mathrm{hrs}$ after cell inoculation were $2 \times 10^{6} \mathrm{CFU} / \mathrm{g}$ for both $S$. Enteritidis and $S$. Typhimurium.

A change in odor measured at different incubation times might be explained by activities of the cells. At the initial stage of the cell growth, small amount of chemical is generated by low cell activity. As cell activity increases, more volatile compounds were produced and such a difference between Salmonella contaminated beef odor and uncontaminated beef odor in terms of peaks was increased. But the amount of produced chemicals was not proportional to the incubation time. The result was similar to that of other researcher (Tahir and Alocilja, 2003), and the reason is that the volatile compounds were diminishing over time because of deteriorated growth environment

Qualitative analysis of the volatile organic compound isolated from the headspace of $S$. Typhimurium was performed with a mass spectrometer. Compounds identified from the isolates are listed in Table 1. Identified compounds were mostly alcohols. Ethanol $(10.2 \%)$ was the main compound, followed by 2-propanamine (5.2\%), 3methyl-1-butanol (4.6\%), ethanedioic acid (3.2\%), 1decanol (2.7\%), ethyl aminopropionate (1.6\%), 2-deuterobutane (1.3\%), 1-dodecanol (1.2\%), 2-methyl-1-butanol $(1.0 \%), 1$-octanol $(0.6 \%)$, and butyl acetate $(0.3 \%)$. Arnold and Senter (1998) analyzed volatile compounds produced by various species of bacteria with SPME GCMS. In their research, gas chromatograms of the VOCs isolated from the headspace of $S$. Enteritidis, Escherichia coli, Listeria monocytogenes, Klebsiella pneumoniae,

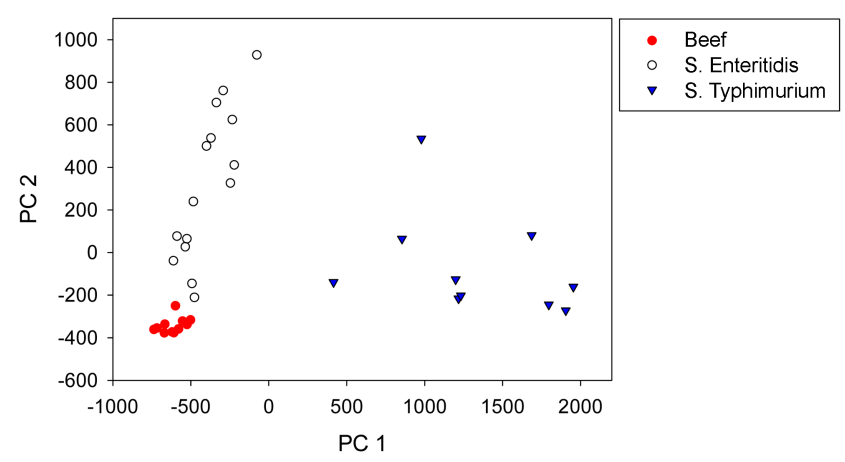

Fig. 6. PCA score plot for the classification of $S$. Enteritidis and $S$. Typhimurium after $4 \mathrm{~h}$ of incubation. 
Table 1. Volatile organic compounds isolates from the headspace of $S$. Typhimurium cultures by SPME (DVB/ CAR/PDMS)

\begin{tabular}{cccc}
\hline \hline Peak & RT & Area (\%) & Compounds \\
\hline 1 & 2.20 & 3.2 & Ethanedioic acid \\
2 & 2.27 & 10.2 & Ethanol \\
3 & 2.40 & 5.2 & 2-Propanamine \\
4 & 2.64 & 1.6 & Ethyl aminopropionate \\
5 & 2.76 & 1.3 & 2-Deuterobutane \\
6 & 5.58 & 4.6 & 3-Methyl-1-butanol \\
7 & 5.69 & 1.0 & 2-Methyl-1-butanol \\
8 & 6.08 & 0.3 & Butyl acetate \\
9 & 18.80 & 0.6 & 1-Octanol \\
10 & 26.80 & 2.7 & 1-Decanol \\
11 & 34.58 & 1.2 & 1-Dodecanol \\
\hline
\end{tabular}

Enterobacter cloacae, Pseudomonas aeruginosa, and Acinetobacter calcoaceticus. Results of Arnold and Senter's study shows that dominant compound of the VOCs isolated from the headspace of $S$. Enteritidis, Escherichia coli, Listeria monocytogenes, Klebsiella pneumoniae, Enterobacter cloacae was also ethanol. Other compounds isolated from the headspace of $S$. Enteritidis were similar to the compounds isolated from the headspace of $S$. Typhimurium in this research. Those compounds were decanol (3.6\%), dodecanol (3.2\%), 3methyo-1-butanol (3.0\%), tetradecanol (2.7\%), 1propanol (2.6\%), cis-7-tetradecene-1-ol (2.2\%), octanol (1.6\%), and 9-decene-1-ol (0.6\%). Other species were distinguished by the production of different compounds such as 3-methyl butanal for Listeria monocytogenes and indole for $E$. coli.

The frequent outbreaks of foodborne illness demand rapid detection of foodborne pathogens. Conventional methods for pathogen detection and identification are labor-intensive and take days to complete. Electronic nose technologies have shown great potential for the rapid detection of odor changes related to food safety. A SAW detector-based electronic nose was used to determine safety of beef by analyzing the odors. Detection of Salmonella contamination of beef was possible by odor analysis. Salmonella contamination of the beef could be classified by PCA after $4 \mathrm{~h}$ of incubation. The classification of Salmonella contamination was mainly described by the first principal component (PC1). The simplicity and speed of the electronic nose may provide potentials for rapid determination of various foodborne pathogens. Further development of this detection technique will make a significant improvement on food safety programs in the future.

\section{Acknowledgment}

This research was supported by the National Academy of Agricultural Science, Rural Development Administration, Korea.

\section{References}

1. Arnold, J. W. and Senter, S. D. (1998) Use of digital aroma technology and SPME GC-MS to compare volatile compounds produced by bacteria isolated from processed poultry. J. Sci. Food Agric. 78, 343-348.

2. Barbri, N. El., Llobet, E., Bari, N. El., Correig, X., and Bouchikhi, B. (2008) Application of a portable electronic nose system to assess the freshness of Moroccan sardines. Materials Sci. Eng. C. 28, 666-670.

3. Blixt, Y. and Borch, E. (1999) Using an electronic nose for determining the spoilage of vacuum-packaged beef. Int. J. Food Microbiol. 46, 123-134.

4. Dutta, R., Hines, E. L., Gardner, J. W., and Boilot, P. (2002) Bacteria classification using Cyranose 320 electronic nose. Biomed. Eng. 1, 1-7.

5. Grigioni, G. M., Margaria, C. A., Pensel, N. A., Sanchez, G., and Vaudagna, S. R. (2000) Warmed-over flavour analysis in low temperature-long time processed meat by an "electronic nose". Meat Sci. 56, 221-228.

6. Keshri, G., Magan, N., and Voysey, P. (1998) Use of an electronic nose for early detection and differentiation between spoilage fungi. Lett. Appl. Microbiol. 27, 261-264.

7. McEntegart, C. M., Penrose, W. R., Strathmann, S., and Stetter, J. R. (2000) Detection and discrimination of coliform bacteria with gas sensor arrays. Sensor. Actuat. B. 70, 170176.

8. Natale, C. D., Olafsdottir, G., Einarsson, S., Martinelli, E., Paolesse, R., and D'Amico, A. (2001) Comparison and integration of different electronic noses for freshness evaluation of cod-fish fillets. Sensor. Actuat. B. 77, 572-578.

9. O'Connell, M., Valdora, G., Peltzer, G., and Hegri, R. M. (2001) A practical approach for fish freshness determinations using a portable nose. Sensor. Actuat. B. 80, 149-154.

10. Rossi, V., Garcia, C., Talon, R., Denoyer, C., and Berdague, J. L. (1996) Rapid discrimination of meat products and bacterial strains using semiconductor gas sensors. Sensor. Actuat. B. 37, 43-48.

11. Rossi, V., Talon, R., and Berdague, J. L. (1995) Rapid discrimination of Micrococcaceae species using semiconductor gas sensors. J. Microbiol. Methods 24, 183-190.

12. Storey, R. M., Davis, H. K., Owen, D., and Moore, L. (1984) Rapid approximate estimation of volatile amines in fish. $J$. Fd. Technol. 19, 1-10.

13. Tahir, Z. M. and Alocilja, E. C. (2003) A conductometric biosensor for biosecurity. Biosens Bioelectron. 18, 813-819.

(Received 2009.6.26/Revised 2010.3.9/Accepted 2010.3.11) 